\title{
Relapsing cytomegalovirus infection in solid organ transplant recipients
}

\author{
A. Shanahan, P.N. Malani, D.R. Kaul. Relapsing cytomegalovirus \\ infection in solid organ transplant recipients. \\ Transpl Infect Dis 2009: 11: 513-518. All rights reserved
}

\begin{abstract}
Efforts to prevent relapsed cy tomegalovirus (CMV) disease among solid organ transplant (SOT) recipients present clinical challenges. Historically, SOT recipients treated with short courses of ganciclovir, without documented clearance of viremia, had relapse rates of $23-33 \%$. Current treatment often includes much longer courses of valganciclovir, and persistence of viremia at the end of treatment is rare. We sought to determine the rate and risk factors for relapse under those treatment conditions. Records of 1760 SOT recipients from January 2003 to June 2007 were reviewed; 105 cases of CMV viremia were identified. Relapse occurred in 20/105 (19\%); 50\% had end-organ disease at the time of relapse. Most patients received approximately 3 months of valganciclovir. Clearance of viremia was documented in 19/20 patients with relapse. Multivariable analysis identified receipt of a thoracic organ and diabetes mellitus as risk factors for relapse. Despite long treatment courses with valganciclovir and documented clearance of viremia, CMV relapse remains common among SOT recipients. Better understanding of the epidemiology of CMV among SOT recipients and validation of risk factors for disease relapse should be the focus of future prospective trials. Such trials should include different treatment durations and extended monitoring for relapse.
\end{abstract}

\author{
A. Shanahan ${ }^{1}$, P.N. Malani ${ }^{2,3,4,5}$, D.R. Kaul ${ }^{2}$ \\ ${ }^{1}$ Department of Internal Medicine, ${ }^{2}$ Division of Infectious \\ Diseases, ${ }^{3}$ Geriatric Medicine, University of Michigan \\ Medical School, Ann Arbor, Michigan, USA, ${ }^{4}$ Veterans Affairs \\ Ann Arbor Healthcare System, ${ }^{5}$ Geriatric Research Education \\ and Clinical Center (GRECC), Ann Arbor, Michigan, USA
}

\author{
Key words: CMV infection; cytomegalovirus; solid organ \\ transplantation; CMV relapse \\ Correspondence to: \\ Daniel R. Kaul, MD, Division of Infectious Diseases, \\ University of Michigan Medical Center, 3120 Taubman Center, \\ P0 Box 5378, Ann Arbor, MI 48109-5378, USA \\ Tel: + 17349368183 \\ Fax: +17349362737 \\ E-mail: kauld@umich.edu
}

Received 5 December 2008, revised 27 March, 21 April 2009, accepted for publication 24 April 2009

DOI: $10.1111 /$ j.1399-3062.2009.00443.x

Transpl Infect Dis 2009: 11: $513-518$
During the past decade, improvements have occurred in the management of cytomegalovirus (CMV) after solid organ transplantation (SOT). The availability of quantitative whole blood or plasma-based assays (e.g., real-time polymerase chain reaction [PCR] or antigenemia assays) has replaced culture-based assays and improved our ability to diagnose CMV infection after SOTand monitor response to treatment (1). These assays may also be used as part of a preemptive monitoring strategy to prevent CMV disease (2). Further, the availability of oral valganciclovir has allowed extended treatment or prophylaxis courses that were previously much less convenient with intravenous (IV) ganciclovir or the older less bioavailable oral ganciclovir (2).

Despite these advances, CMV remains the leading viral cause of morbidity and mortality following SOT, and a number of major questions remain regarding the optimal management of $\mathrm{CMV}$ infection. In particular, the appropriate duration of therapy for CMV disease has not been well studied. In previous studies of recurrent CMV disease, relapse after treatment was observed in $23-33 \%$ of cases (3-8); in most reports a short course (2-3 weeks) of treatment was used $(3-5,7)$. Relapse has been attributed to incomplete suppression of viremia at the conclusion of treatment (9), and this has led to the recommendation that treatment be continued until viremia resolves or for 1 week after viremia resolves $(9,10)$. No randomized studies, however, have addressed this question.

In our center, even longer courses of antiviral treatment (generally 90 days) and monitoring of CMV viremia during treatment are typically used. Despite these practices, we noted frequent relapse of CMV infection or disease. Thus, we sought to determine the incidence and risk factors for relapse of CMV infection and disease after SOT among patients treated with prolonged courses of valganciclovir with documented resolution of viremia before the completion of treatment. 


\section{Methods}

\section{Patients and setting}

The University of Michigan Health System is an 850-bed tertiary care facility with an active transplantation program. The electronic medical record (CareWeb) of all patients who received an SOT from January 2003 to June 2007 was reviewed. During that period, a total of 1760 transplants were performed; 1019 kidney, 82 pancreas or kidney-pancreas, 400 liver, 115 lung, and 144 heart transplants. Those patients with at least 1 CMV PCR > 1000 copies/mL (Roche Amplicor CMV Monitor Test, Branchburg, New Jersey, USA) were included in the study.

CMV prophylactic strategies varied based on time of transplant and organ transplanted, but all patients at risk for CMV disease (donor positive [D + ] and/or recipient positive $[\mathrm{R}+]$ ) received prophylaxis with valganciclovir for 90 days. After 2005, high-risk kidney transplants (D +, R - ) received 6 months of prophylaxis; all other SOT recipients continued to receive 90 days of valganciclovir, although total duration of prophylaxis varied somewhat based on the discretion of the treating physician. Liver transplants received valganciclovir, although it is not Food and Drug Administration approved for this indication. The ganciclovir and valganciclovir dose was adjusted for renal function.

Immunosuppression protocols varied, but recipients of lung and liver transplants did not receive induction immunosuppression. Heart transplant patients with baseline renal dysfunction or those considered to be at risk for renal dysfunction (e.g., prolonged operative time) and at high immunologic risk for rejection received induction immunosuppression with muromonab-CD3 (OKT3) $2.5 \mathrm{mg}$ IV daily $\times 3-10$ days or thymoglobulin $100-125 \mathrm{mg} \times 2$ days then $50 \mathrm{mg} \times 6-10$ days. High-risk kidney transplant recipients (African-American, previous transplant, panel reactive antibodies $>30 \%$, unrelated living donor) received thymoglobulin. Low-risk patients with delayed graft function received basiliximab. For maintenance immunosuppression, most renal, heart, and lung transplant recipients received mycophenolate mofetil, cyclosporine or tacrolimus, and prednisone. In most liver recipients, immunosuppression was reduced over 2-3 months to tacrolimus alone. This study was approved by the University of Michigan Health System Institutional Review Board.

\section{Data collection and definitions}

Transplant recipients with at least 1 CMV PCR > 1000 copies/mL were identified as having CMV infection and included in the study. Patients with CMV disease but without viremia were not included. CMV disease was de- fined as patients with CMV viremia and a febrile syndrome without an alternative explanation, or patients with CMV viremia and clinical or histological evidence of end-organ involvement. No routine monitoring strategy for CMV infection was used, and all CMV PCR tests and other studies were obtained at the discretion of treating physicians.

Relapsed CMV infection was defined as CMV viremia ( $>1000$ copies $/ \mathrm{mL}$ ) recurring within 100 days of completing treatment. Relapsed CMV disease utilized the same definitions as in the preceding paragraph. Demographic and clinical data were collected in all patients with CMV infection or disease including prednisone dose, donor and recipient CMV serologies, co-morbidities, rejection therapy, duration of CMV prophylaxis, whether or not viremia was documented to have cleared, and time to recurrence. CMV treatment data including duration of therapy were also collected.

\section{CMV treatment protocols}

Renal transplant patients with CMV disease received oral valganciclovir $900 \mathrm{mg}$ orally twice a day for 21 days followed by $900 \mathrm{mg}$ daily to complete a total 90 -day treatment course. The dose was adjusted as appropriate for renal function. Patients unable to reliably take oral valganciclovir received IV ganciclovir $(5 \mathrm{mg} / \mathrm{kg}$ twice a day, adjusted for renal function) until able to take oral therapy. Duration of treatment and dosing for recipients of other transplants was based on the discretion of the treating physician. For all patients, CMV monitoring both during treatment and after conclusion of treatment varied, based on the treating physician and clinical circumstances.

\section{Data analysis}

Risk factors for relapse of infection or disease were calculated. A separate calculation of risk factors was performed and limited to patients who relapsed with CMV disease (relapse of infection alone was excluded). Univariate analysis using $t$-tests or Wilcoxon's rank-sum performed for continuous variables and chi-square or Fisher's exact test for categorical variables identified risk factors for CMV relapse. Two-tailed $P$-value $\leq 0.05$ was considered statistically significant. Crude odds ratio (OR) and $95 \%$ confidence interval $(\mathrm{CI})$ were calculated for categorical variables. Crude ORs for continuous variables were obtained using simple logistic regression. Variables that were significant to a $P$-value of 0.20 as well as those variables that had a priori clinical significance were then analyzed using multivariate logistic regression modeling. All statistical analysis was performed using SAS 9.1 (SAS Institute Inc., Cary, North Carolina, USA). 


\section{Results}

We identified 105 patients with CMV viremia among whom $20(19 \%)$ demonstrated relapse. Of the patients with relapse, 10/20 (50\%) had CMV disease and 10/20 (50\%) had CMV infection without disease. In 19/20 (95\%) of relapsed cases, viremia was documented to have cleared at the completion of antiviral therapy. In cases without relapse, 59/85 $(69 \%)$ cleared viremia, $2 / 85(2 \%)$ did not clear viremia, and in $24 / 85(28 \%)$ further studies were not obtained to document clearance. These patients did not have clinical symp- toms prompting a repeat CMV PCR. Four of these 24 patients died; no others were lost to follow up. Risk factors for relapsed infection identified by univariate analysis included advancing age, diabetes mellitus, and receipt of a chest as opposed to an abdominal organ (Table 1). Relapse rates were highest among recipients of heart transplants 6 / $16(38 \%)$ and lowest for kidney transplant recipients $6 / 51(11 \%)$ (Table 1). In multivariate analysis, diabetes mellitus (adjusted OR 4.1, 95\% CI [1.3-13]P=0.02) and receipt of thoracic organ (adjusted OR 3.6, 95\% CI [1.2-10.6] $P=0.02$ ) were significant (Table 2). Duration of treatment for the initial episode of CMV viremia did not differ signifi-

Characteristics of solid organ transplant recipients with cytomegalovirus (CMV) viremia

\begin{tabular}{|c|c|c|c|c|}
\hline \multirow[b]{2}{*}{ Characteristic } & \multirow{2}{*}{$\frac{\text { No relapse }}{85(81 \%)}$} & \multirow{2}{*}{$\frac{\text { Relapse }}{20(19 \%)}$} & \multirow[b]{2}{*}{$\mathrm{OR}(95 \% \mathrm{Cl})$} & \multirow[b]{2}{*}{$P$-value } \\
\hline & & & & \\
\hline \multicolumn{5}{|l|}{ Demographics } \\
\hline Age $^{1}$ & 48 (range $21-73$ ) & 52 (range 36-69) & $1.05(1.0-1.1)$ & 0.05 \\
\hline Female & $30(35 \%)$ & $8(40 \%)$ & $1.2(0.5-3.3)$ & 0.90 \\
\hline White race & $71(84 \%)$ & $20(100 \%)$ & $8.3(0.5-145)$ & 0.08 \\
\hline \multicolumn{5}{|l|}{ Co-morbidities } \\
\hline Diabetes & $38(45 \%)$ & $14(70 \%)$ & $3.7(1.2-11.1)$ & 0.01 \\
\hline Renal insufficiency (serum creatinine $>1.5$ ) & $23(27 \%)$ & $6(30 \%)$ & $1.6(0.4-3.4)$ & 0.79 \\
\hline \multicolumn{5}{|l|}{ Transplanted organ } \\
\hline Renal & 51 & $6(11 \%)$ & $0.3(0.1-0.8)$ & 0.03 \\
\hline Renal and pancreas & 3 & $1(25 \%)$ & $1.4(0.14-14.6)$ & 0.99 \\
\hline Pancreas alone & 2 & $1(33 \%)$ & $2.2(0.2-25.3)$ & 0.99 \\
\hline Liver & 9 & $2(18 \%)$ & $0.9(0.2-4.7)$ & 0.99 \\
\hline Lung & 10 & $4(29 \%)$ & $1.9(0.5-6.7)$ & 0.99 \\
\hline Heart & 10 & $6(38 \%)$ & $3.2(1.0-10.3)$ & 0.08 \\
\hline Chest organ & 20 & $10(33 \%)$ & $3.3(1.2-8.9)$ & 0.02 \\
\hline Abdominal organ & 65 & $10(13 \%)$ & $0.3(0.1-0.8)$ & 0.02 \\
\hline \multicolumn{5}{|l|}{ CMV status } \\
\hline High risk & $58(68 \%)$ & $13(65 \%)$ & $1.2(0.4-3.6)$ & 0.99 \\
\hline Recipient positive & $24(28 \%)$ & $5(25 \%)$ & $0.9(0.3-2.6)$ & 0.99 \\
\hline Donor and recipient negative & $2(2 \%)$ & 0 & $0.8(0.04-17.6)$ & 0.99 \\
\hline CMV status unknown & $1(1 \%)$ & $2(10 \%)$ & $9.3(0.8-109)$ & 0.18 \\
\hline \multicolumn{5}{|l|}{ Induction immunosuppression } \\
\hline Cell depleting induction & $37(44 \%)$ & $11(55 \%)$ & $1.6(0.6-4.2)$ & 0.50 \\
\hline IL-2R antagonist & $23(27 \%)$ & $4(20 \%)$ & $0.7(0.2-2.2)$ & 0.74 \\
\hline No induction & 25 (29\%) & $5(25 \%)$ & $0.8(0.26-2.4)$ & 0.93 \\
\hline \multicolumn{5}{|l|}{ Maintenance immunosuppression } \\
\hline Prednisone dose at diagnosis (mg) & 10 (range 0-60) & 10 (range 0-40) & & 0.94 \\
\hline Prednisone dose at completion of treatment (mg) & 10 (range 0-20) & 10 (range 0-15) & & 0.77 \\
\hline Absolute lymphocyte count at diagnosis & $0.84 \pm 1.0$ & $0.73 \pm 1.3$ & & 0.70 \\
\hline
\end{tabular}


Table 1 Continued

\begin{tabular}{|c|c|c|c|c|}
\hline \multirow[b]{2}{*}{ Characteristic } & \multirow{2}{*}{$\frac{\text { No relapse }}{85(81 \%)}$} & \multirow{2}{*}{$\frac{\text { Relapse }}{20(19 \%)}$} & \multirow[b]{2}{*}{$\mathrm{OR}(95 \% \mathrm{Cl})$} & \multirow[b]{2}{*}{$P$-value } \\
\hline & & & & \\
\hline \multicolumn{5}{|l|}{ Duration of CMV prophylaxis } \\
\hline None & $4(5 \%)$ & 0 & $0.4(0.02-8.5)$ & 0.85 \\
\hline$<90$ days & $4(5 \%)$ & $3(15 \%)$ & $3.5(0.7-17.4)$ & 0.99 \\
\hline $90-130$ days & $63(74 \%)$ & $13(65 \%)$ & $1.0(0.4-2.2)$ & 0.87 \\
\hline $135-180$ days & $9(11 \%)$ & $4(20 \%)$ & $2.1(0.6-7.7)$ & 0.44 \\
\hline$>180$ days & $1(1 \%)$ & 0 & $1.3(0.05-35)$ & 0.99 \\
\hline Unknown & $4(5 \%)$ & 0 & $0.4(0.02-8.5)$ & 0.85 \\
\hline Days post transplant to time of CMV diagnosis (median) ${ }^{2}$ & 167 & 180 & & 0.29 \\
\hline Clinical evidence for end organ disease & $52(61 \%)$ & $16(80 \%)$ & $0.42(0.16-1.1)$ & 0.14 \\
\hline Peak CMV copies $/ \mathrm{mL}$ (median) ${ }^{2}$ & 22,700 (range $10,400-100,000$ ) & 23,200 (range 1270-100,000) & & 0.21 \\
\hline Peak CMV copied/mL $>100,000$ & $15(18 \%)$ & $4(20 \%)$ & $1.2(0.34-4.0)$ & 0.99 \\
\hline Treatment duration (days) & $93.8 \pm 77$ & $84.2 \pm 54$ & & 0.59 \\
\hline
\end{tabular}

\section{Table 1}

cantly between recipients of different organs and was as follows: heart median 83 days (range 30-360), kidney median 90 days (range 14-205), liver median 38 days (range 4187), and lung median 104 days (range 14-365).

Receipt of rejection therapy was not different between patients with relapse $(2 / 20,10 \%)$ and without relapse $(9 /$ $85,11 \%)$. Although the number of patients with high-risk serostatus $(\mathrm{D}+, \mathrm{R}-)$ ) was similar among abdominal and thoracic organ recipients $(60 \%$ thoracic and $74 \%$ abdominal), thoracic organ recipients with high-risk serostatus were much more likely to develop CMV relapse (8/18 [44\%] versus 5/53 [9\%], OR 7.7 [2.1-28.4], $P=0.005$ ).

Multivariable analysis of the association of select patient characteristics on relapsed cytomegalovirus (CMV) infection among solid organ transplant recipients

\begin{tabular}{|c|c|c|}
\hline Characteristic & aOR $(95 \% \mathrm{Cl})^{1}$ & $P$-value \\
\hline $\mathrm{Age}^{2}$ & $1.05(1.0-1.1)$ & 0.07 \\
\hline Diabetes mellitus & $4.1(1.3-13)$ & 0.02 \\
\hline Receipt of thoracic organ & $3.6(1.2-10.6)$ & 0.02 \\
\hline \multicolumn{3}{|c|}{$\begin{array}{l}{ }^{1} \text { Adjusted odds ratios }(\mathrm{aOR}) \text { and } 95 \% \text { confidence intervals }(\mathrm{Cl}) \text { are basec } \\
\text { on a multivariable logistic regression adjusting for age, diabetes mellitus } \\
\text { receipt of a thoracic organ, high-risk } \mathrm{CMV} \text { serostatus, and prednisone } \\
\text { dose at diagnosis of } \mathrm{CMV} \text {. } \\
{ }^{2} \text { OR for age reported for } 1 \text {-year unit. }\end{array}$} \\
\hline
\end{tabular}

Table 2
A univariate analysis of the 10 patients with relapsed disease (but excluding those with infection without disease) was performed using the same variables. The presence of diabetes mellitus $(8 / 10$ [80\%] versus $45 / 95$ [47\%], OR 4.4, $95 \% \mathrm{CI}[0.9-22] P=0.09$ ) was not significant, and age was not a risk factor for relapse. Recipients of renal transplants (without pancreas) were less likely to relapse than recipients of other organs $(2 / 10[20 \%$ ] versus $55 / 95$ [58\%] OR 0.2 $95 \%$ CI $[0.04-0.9] P=0.05$ ), and recipients of chest organs were more likely to relapse $(6 / 10[60 \%]$ versus $24 / 95$ [25\%], OR 4.4, 95\% CI [1.2-17.1] $P=0.05$ ). Of the 10 patients with relapsed CMV disease, CMV syndrome was present in 4, enteritis in 3, and pneumonitis in 3. Among all relapsed patients, the median CMV viral load at time of relapse was 6208 copies $/ \mathrm{mL}$, and relapse occurred a mean of 38 days and a median of 60 days after completing initial treatment (range 7-98 days). No ganciclovir resistance was noted among relapsed patients, and all patients were treated with either ganciclovir or valganciclovir.

\section{Discussion}

Despite extended courses of treatment with oral valganciclovir and documented resolution of viremia in all but 1 case, a relatively high rate of relapse of CMV infection was observed among SOT recipients. Longer treatment of 
CMV viremia or disease and high-risk serostatus did not appear to be associated with a reduced risk of relapse. Recipients of thoracic organs were at higher risk of relapse than recipients of abdominal organs, and high-risk serostatus $(\mathrm{D}+, \mathrm{R}-)$ recipients of chest organs experienced a particularly high rate of relapse. Half of relapsed cases had CMV disease at the time of relapse, and half had CMV infection without disease.

Very few studies have addressed relapsing CMV infection among SOT recipients, with reported relapse rates of 23-33\% (Table 3). The majority of these studies were conducted before the availability of highly bioavailable oral valganciclovir, which makes longer treatment courses more feasible. In 4 of the 6 studies in Table 3 , treatment consisted of short courses of IV ganciclovir, and viremia was commonly present (or not proven to have resolved) at the time of completion of treatment $(3-5,7)$. The overall rate of CMV relapse observed in our study $(19 \%)$ is only marginally lower. If the rate among kidney transplants alone is compared, however, our rate of 6/51 $(11 \%)$ shows a clear trend toward being lower than the $25 \%$ relapse rate observed by Franco et al. (4).

Two of the 6 studies prescribed longer courses of treatment. Turgeon et al. (8) followed a 2-3-week course of IVganciclovir with 2-3 months of the older, less bioavailable oral preparation of ganciclovir, but relapse remained high at $27 \%$. In the recent report from Levitsky et al. (6), 28 patients received extended treatment (median 119 days IV ga- nciclovir followed by oral valganciclovir or median 104 days valganciclovir alone), and an even higher relapse rate of $33 \%$ was reported. The majority of recurrences, however, were asymptomatic and not treated with additional antiviral therapy. By contrast, among our patients, $50 \%$ had endorgan disease at the time of relapse. In our center, monitoring for CMV viremia after completion of treatment varied based on treating physician preference. This variability may account for differences in the rate of asymptomatic CMV infection and CMV disease observed among patients with relapse.

Risk factors for relapsed disease have varied across previous studies (Table 3 ). Two studies identify failure to clear viremia as a risk factor for relapse $(4,5)$, and it is certainly biologically plausible to expect that CMV would be more likely to relapse among patients with viremia after completing treatment. Thus, it is somewhat surprising that, despite a relatively high recurrence rate, virtually all patients with relapse in our study had resolution of viremia at completion of primary therapy.

In addition, our study identified receipt of a chest organ as a risk factor for relapse. In particular, high-risk serostatus thoracic organ recipients had a $44 \%$ relapse rate. Lung transplant recipients in particular are known to be at higher risk for CMV disease (10), and perhaps the same factors that put them at risk for initial disease account for the increased risk for relapsed disease. We also noted that the initial prednisone dose at the time of initial CMV diagnosis

Review of published studies of relapsing cytomegalovirus (CMV) disease in solid organ transplant (SOT) recipients

\begin{tabular}{|c|c|c|c|c|c|}
\hline Study & Population & Treatment & Relapse rate & Risk factors for relapse & Comments \\
\hline Franco et al. (4) & $\begin{array}{l}49 \text { renal transplant } \\
\text { recipients }\end{array}$ & 2 weeks of IV ganciclovir & $12 / 49(24.5 \%)$ & $\begin{array}{l}\text { Failure to clear viremia } \\
\text { Acute rejection }\end{array}$ & \\
\hline Humar et al. (5) & $\begin{array}{l}52 \text { SOT recipients (liver } \\
35 \text {, kidney } 7 \text {, lung } 7 \text {, } \\
\text { other } 3 \text { ) }\end{array}$ & 2-3 weeks of IV ganciclovir & $12 / 52(23.1 \%)$ & $\begin{array}{l}\text { Failure to clear viremia } \\
\text { Tıme to clearance } \\
\text { Slow decrease of viral load in first } \\
\text { week of treatment }\end{array}$ & \\
\hline Sia et al. (7) & $\begin{array}{l}24 \text { SOT recipients } \\
\text { (heart, liver, kidney) }\end{array}$ & 2 weeks of IV gancicloivir & $8 / 33(33 \%)$ & $\begin{array}{l}\text { High-risk serostatus } \\
\text { Higher pre- and post-treatment } \\
\text { viral loads }\end{array}$ & $\begin{array}{l}25 \% \text { had persistent } \\
\text { viremia after } 14 \text { days } \\
\text { treatment }\end{array}$ \\
\hline Turgeon et al. (8) & $\begin{array}{l}19 \text { kidney, } 18 \text { liver } \\
\text { transplant recipients }\end{array}$ & $\begin{array}{l}2-3 \text { weeks of IV ganciclovir, } \\
\text { then } 2-3 \text { months of oral } \\
\text { ganciclovir }(2 \mathrm{~g} / \text { day })\end{array}$ & $10 / 37(27 \%)$ & $\begin{array}{l}\text { High-risk serostatus } \\
\text { Higher peak antigenemia value } \\
\text { during treatment }\end{array}$ & \\
\hline Falagas et al. (3) & $\begin{array}{l}41 \text { liver transplant } \\
\text { recipients }\end{array}$ & $8+$ days of IV ganciclovir & $11 / 41(27 \%)$ & $\begin{array}{l}\text { Presence of multiorgan disease } \\
\text { CMV pneumonia }\end{array}$ & \\
\hline Levitsky et al. (6) & $\begin{array}{l}28 \text { SOT (kidney, liver, } \\
\text { heart, lung; } 6 \text { received } \\
\text { chest organ) }\end{array}$ & $\begin{array}{l}119 \text { days of IV ganciclovir } \\
\text { followed by oral valgan or } 104 \\
\text { days of valgancyclovir } \\
\text { alone }\end{array}$ & $11 / 28(33 \%)$ & None & $\begin{array}{l}\text { Majority of recurrences } \\
\text { were asymptomatic } \\
\text { and not treated }\end{array}$ \\
\hline
\end{tabular}

Table 3 
is higher among patients with thoracic organs $(13.4 \pm 15$ versus $9.7 \pm 2.4 \mathrm{mg}, P=0.03$ ). In the cohort as a whole, however, prednisone dose did not show significance in either univariate analysis or multivariate regression models of risk factors for relapse. The dose at the end of therapy was not different between the groups.

In the univariate analysis, increasing age was also identified as a risk factor, but was of only borderline significance in the multivariate analysis. Cell-mediated immunity is known to wane with increasing age (11), and this may have accounted for this association. Impaired immunity may also explain the identification of diabetes mellitus as a risk factor for relapse. These risk factors deserve additional consideration in future studies.

Our study has a number of important limitations. Comparison of rates of CMV infection or disease from one study to another are complicated by changes in diagnostic tests available over time as well as center-to-center differences in practice patterns for CMV monitoring after completion of treatment. No set protocol for monitoring is established at our institution, thus the approach varied depending on physician preference. These practices may result in an ascertainment bias that may affect the number of cases of relapse diagnosed. For example, routine monitoring for CMV viremia or antigenemia would likely identify some patients who might resolve their viremia without further treatment. In addition, the management of CMV infection and disease (including duration of treatment) varied considerably by type of organ as well as from patient to patient transplanted with the same organ. Such differences may have accounted for the higher risk of relapse observed in recipients of chest organs. In order to account for this, we also analyzed by the endpoint of CMV disease, as ascertainment bias would be expected to be less of factor in patients presenting with clinical symptoms suggestive of CMV disease. In this analysis, recipients of chest organs were at higher risk of relapse than renal recipients, but diabetes mellitus and age were not significant. Finally, we only studied episodes of CMV viremia, but did not attempt to identify patients with CMV disease who did not have viremia.

Our results suggest that, despite long treatment courses with oral valganciclovir and resolution of viremia, CMV relapse remains relatively common among SOT recipients. Recipients of abdominal organs, however, may be at lower risk than recipients of thoracic organs. Patients at higher risk of relapse (e.g., older recipients of thoracic organs or patients with diabetes) may benefit from more intensive monitoring for relapse. Despite the limitations of our study design, the $19 \%$ CMV relapse rate is notable. Future pro- spective, randomized trials of different durations of treatment (e.g., 1 versus 4 weeks after resolution of viremia) are warranted. Another strategy that has been suggested to individualize prophylaxis involves the use of patient-specific markers of CMV cell-mediated immunity (12). A similar strategy could be tried to predict risk of relapse. In any case, such trials should include extended monitoring for relapse (up to 100 days) and a quantitative threshold (e.g., $>5,000$ copies/mL on 2 separate measurements) for treating relapse in patients with asymptomatic viremia.

\section{References}

1. AlliceT, Enrietto M, Pittaluga F, et al. Quantitation of cytomegalovirus DNA by real-time polymerase chain reaction in peripheral blood specimens of patients with solid organ transplants: comparison with end-point PCR and pp65 antigen test. J Med Virol 2006; 78 (7): 915-922.

2. Lopau K, Greser A, Wanner C. Efficacy and safety of preemptive antiCMV therapy with valganciclovir after kidney transplantation. Clin Transplant 2007; 21 (1): 80-85.

3. Falagas ME, Snydman DR, Griffith J, Werner BG, Freeman R, Rohrer R. Clinical and epidemiological predictors of recurrent cytomegalovirus disease in orthotopic liver transplant recipients. Clin Infect Dis 1997; 25 (2): 314-317.

4. Franco A, Serrano R, Gimeno A, et al. [Evaluation of viral load and antigenemia as markers for relapse cytomegalovirus infection in renal transplant recipients.] Nefrología 2007; 27 (2): 202-208.

5. Humar A, Kumar D, Boivin G, Caliendo AM. Cytomegalovirus (CMV) virus load kinetics to predict recurrent disease in solid-organ transplant patients with CMV disease. J Infect Dis 2002; 186 (6): 829 833.

6. Levitsky J, Freifeld AG, Puumala S, et al. Cytomegalovirus viremia in solid organ transplantation: does the initial viral load correlate with risk factors and outcomes? Clin Transplant 2008; 22 (2): 222-228.

7. Sia IG, Wilson JA, Groettum CM, Espy MJ, Smith TF, Paya CV. Cytomegalovirus (CMV) DNA load predicts relapsing CMV infection after solid organ transplantation. J Infect Dis 2000; 181 (2): 717-720.

8. Turgeon N, Fishman JA, Doran M, et al. Prevention of recurrent cytomegalovirus disease in renal and liver transplant recipients: effect of oral ganciclovir. Transpl Infect Dis 2000; 2 (1): 2-10.

9. Preiksaitis JK, Brennan DC, Fishman J, Allen U. Canadian society of transplantation consensus workshop on cytomegalovirus management in solid organ transplantation final report. Am J Transplant 2005; 5 (2): 218-227.

10. Cytomegalovirus. Am J Transplant 2005; 4 (Suppl 10): 51-58.

11. Ginaldi L, De Martinis M, D’Ostilio A, Marini L, Loreto MF, Quaglino D. Immunological changes in the elderly. Aging (Milano) 1999; 11 (5): 281-286.

12. Westall GP, Mifsud NA, Kotsimbos T. Linking CMV serostatus to episodes of CMV reactivation following lung transplantation by measuring CMV-specific CD8 + T-cell immunity. Am J Transplant 2008; 8 (8): 1749-1754. 